\title{
乌ో
}

\section{Rydberg-State-Enabled Deceleration and Trapping of Cold Molecules}

\author{
S. D. Hogan, Ch. Seiler, and F. Merkt \\ Laboratorium für Physikalische Chemie, ETH Zürich, CH-8093, Switzerland
}

(Received 6 June 2009; published 14 September 2009)

\begin{abstract}
Hydrogen molecules in selected core-nonpenetrating Rydberg-Stark states have been decelerated from a mean initial velocity of $500 \mathrm{~m} / \mathrm{s}$ to zero velocity in the laboratory frame and loaded into a threedimensional electrostatic trap. Trapping times, measured by pulsed electric field ionization of the trapped molecules, are found to be limited by collisional processes. As Rydberg states can be deexcited to the absolute ground state, the method can be applied to generate cold samples of a wide range of species.
\end{abstract}

DOI: 10.1103/PhysRevLett.103.123001

Considerable efforts are being invested in the development of methods to generate translationally cold $(<1 \mathrm{~K})$ samples of molecules [1-4]. These efforts are driven by the desires to study molecular collisions at very low energies [5-7], to carry out precision spectroscopic measurements $[8,9]$, and to explore the properties of molecular gases close to quantum degeneracy [2-4].

Since laser cooling techniques are not in general applicable to molecules, the low temperatures must be reached by alternative means: Photoassociation of ultracold atoms [10], buffer gas cooling [11], multistage Stark [12] and Zeeman $[13,14]$ deceleration of polar molecules and radicals in supersonic beams, and sympathetic cooling of molecular ions in Coulomb crystals [6,7] have all been exploited. It has also been possible to load and store the cold samples in electrostatic [15,16], electrodynamic [17], magnetoelectrostatic [18], and magnetic traps $[11,19]$ and study their physicochemical properties. Despite these successes, the diversity of species that have been prepared at low temperatures remains limited.

Demonstrated here, with the example of $\mathrm{H}_{2}$, is the single-stage Stark deceleration and electrostatic trapping of translationally cold molecules in Rydberg states initially traveling in supersonic beams. This technique is of particular relevance to species that cannot be laser cooled and have no permanent electric or magnetic dipole moment in their ground state. The method, first suggested by Breeden and Metcalf [20] and Wing [21], and referred to as Rydberg-Stark deceleration in the following, exploits the large electric dipole moments $\left(e a_{0} n^{2}\right.$, i.e., $\sim 3000$ Debye at
PACS numbers: 37.10.De, 32.60.+i, 33.80.Rv, 37.20.+j

$n=30$ ) accessible by photoexcitation of atoms or molecules to Rydberg states in the presence of an external electric field and the forces that result from the interaction of these dipoles with electric field gradients. Such methods have enabled the deflection of Kr beams [22], the partial deceleration of $\mathrm{H}_{2}[23,24]$ and $\mathrm{Ar}[25,26]$ beams, and the reflection [27] and trapping [16,28] of $\mathrm{H}$ atoms. The ability to control the translational motion of Rydberg atoms and molecules is of interest to a wide range of disciplines, with potential applications in experiments on antihydrogen [29], ultracold plasmas [30], and quantum information processing [31].

Although Rydberg-Stark deceleration is in principle applicable to any gas phase species, its application to molecular samples has suffered from two drawbacks: First, the Stark states of nonhydrogenic atoms and molecules undergo avoided crossings for electric field strengths at and beyond the Inglis-Teller limit [32]. At such crossings, low-field-seeking states can be converted to highfield-seeking states limiting the range of usable field strengths to $F<\frac{1}{3 n^{5}}$ (in atomic units). Second, whereas the lifetimes of $n>25$ Rydberg-Stark states of atoms exceed $50 \mu \mathrm{s}$, the lifetimes of such states in molecules are generally limited by predissociation and usually much shorter [33]. Both of these drawbacks have their origin in core-penetrating, low- $\ell$ components of the Stark wave functions and can therefore be overcome simultaneously by eliminating such components using a suitable multiphoton excitation sequence.

In the absence of an external electric field, the excitation sequence chosen for $\mathrm{H}_{2}$

$$
\begin{aligned}
{\left[\left(1 s \sigma_{g}\right)^{1}\left(v^{+}=0, N^{+}=0\right)\right](n f)^{1},\left(v=0, J=3,\left|M_{J}\right|=3\right) } & \stackrel{\cup}{\longleftarrow}\left(1 s \sigma_{g}\right)^{1}\left(3 d \pi_{g}\right)^{1} I^{1} \Pi_{g}\left(v=0, J=2,\left|M_{J}\right|=2\right) \\
& \longleftarrow\left(1 s \sigma_{g}\right)^{1}\left(2 p \sigma_{u}\right)^{1} B^{1} \Sigma_{u}^{+}\left(v=3, J=1,\left|M_{J}\right|=1\right) \\
& \longleftarrow\left(1 s \sigma_{g}\right)^{2} X^{1} \Sigma_{g}^{+}(v=0, J=0),
\end{aligned}
$$

raises, at each step, the value of $\ell, J$, and $\left|M_{J}\right|$ by one. By virtue of the fact that in para- $\mathrm{H}_{2}$ singlet-triplet mixing is negligible below $n=100$ [34], the accessible Rydberg series converging to the $N^{+}=0$ ionic ground state are $\ell=$
$J=\left|M_{J}\right|=3$ Rydberg states. In the presence of a dc electric field at excitation, $\ell$ and $J$ are no longer good quantum numbers. However, provided the direction of propagation of the laser beams employed is parallel to 
the electric field vector, the accessible Rydberg states converging to an $N^{+}=0$ ion core have $\left|M_{J}\right|=\left|m_{\ell}\right|=3$ which precludes any $\ell=0-2$ components in the Stark wave functions and guarantees their nonpenetrating "hydrogenic" character. The sections of the $\left|M_{J}\right|=0$ and $\left|M_{J}\right|=3$ Stark maps displayed in Figs. 1(b) and 1(c) demonstrate the effects of $\left|M_{J}\right|$ on the size of the avoided crossings among the $N^{+}=0, n=22$, and $n=23$ Stark states of $\mathrm{H}_{2}$. These maps were calculated following the matrix diagonalization procedure described in Ref. [24] using quantum defect data for the Rydberg states of $\mathrm{H}_{2}$ [34] and take into account the interactions with $N^{+}=2$ channels.

A schematic view of the experimental setup is presented in Fig. 1(a). A pulsed supersonic beam of $\mathrm{H}_{2}$ seeded in $\mathrm{Kr}$ at a pressure ratio of 2:8, with a mean longitudinal velocity of $500 \mathrm{~m} / \mathrm{s}$, propagates through a skimmer into a set of six electrodes which constitute the photoexcitation region, the Stark decelerator, and the 3D electrostatic trap. At the time of photoexcitation, electrodes 1 and 2 are held at potentials of $\pm V_{\text {trap }}$ with electrodes 3 and 4 at $\mp V_{\text {trap }}$ forming a quadrupole field distribution between these electrodes. Electrodes 5 and 6 are held at $0 \mathrm{~V}$ and close off the trap in the third dimension [16]. Photoexcitation takes place in a region between electrodes 1 and 2 . To restrict the exci-
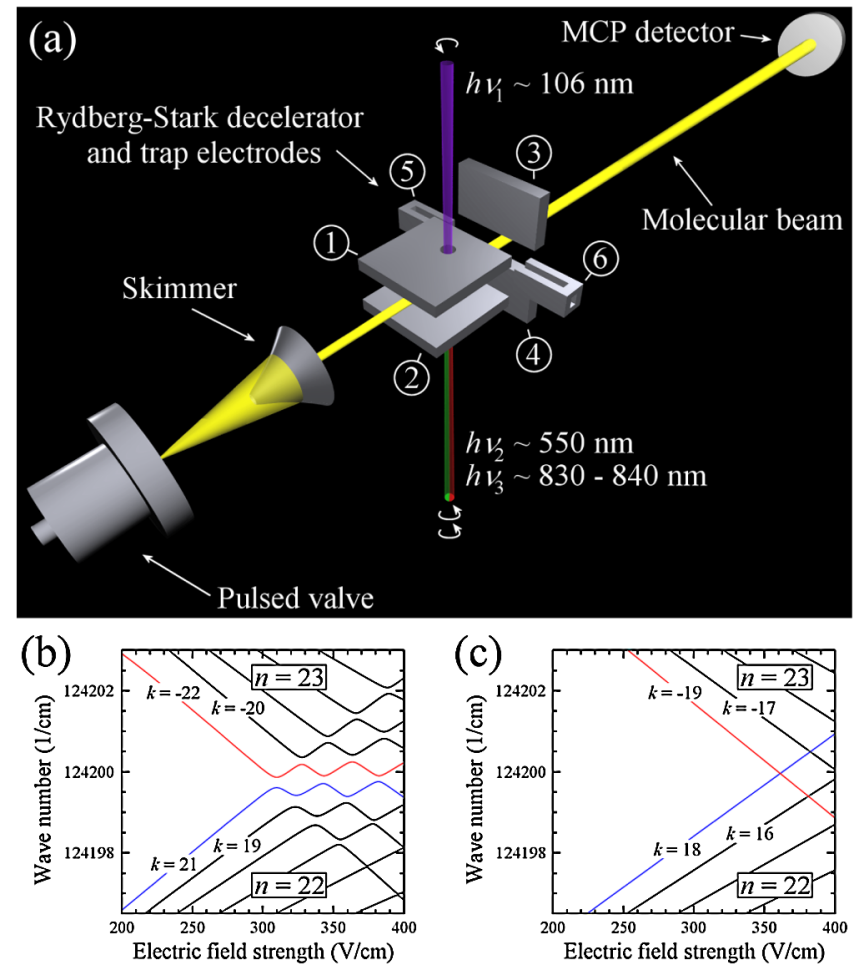

FIG. 1 (color online). (a) Schematic diagram of the experimental setup used to decelerate and trap cold $\mathrm{H}_{2}$ molecules. (b) Avoided crossings between the outermost $n=22$ blueshifted and $n=23$ redshifted Rydberg states of $\mathrm{H}_{2}$ for $\left|M_{J}\right|=0$ and (c) $\left|M_{J}\right|=3$. The Stark states are labeled with the quantum number $k$, which represents the difference between the two parabolic quantum numbers $n_{1}$ and $n_{2}$. tation to $\left|M_{J}\right|=3$ Rydberg-Stark states using three circularly polarized laser beams [see Eq. (1)], all three laser beams must propagate parallel to the dc electric field vector at the position of excitation. To achieve this, $1.5 \mathrm{~mm}$ diameter holes have been drilled through electrodes 1 and 2 as indicated in Fig. 1(a).

The first step of the excitation sequence requires circularly polarized vacuum-ultraviolet (vuv) radiation at $94093.81 \mathrm{~cm}^{-1}(\sim 106 \mathrm{~nm})$ which was generated by resonance-enhanced four-wave mixing in a pulsed Xe jet $\left(\nu_{\mathrm{vuv}}=2 \nu_{1}+\nu_{2}\right)$. The divergent vuv beam was recollimated, separated from the fundamental laser beams, and directed toward the hole in electrode 1 of the decelerator using a toroidal grating. Circular polarization of the vuv radiation was achieved by circularly polarizing the tunable laser beam $\left(\nu_{2}\right)$ in the four-wave-mixing process, using a Glan-Taylor polarizer followed by a Berek compensator, while the two-photon resonant laser beam was linearly polarized. The circular polarization of the vuv radiation was checked spectroscopically and optimized to a purity of better than $90 \%$ by ensuring the disappearance of the $Q(1)$ line of the $I^{1} \Pi_{g} \leftarrow B{ }^{1} \Sigma_{u}^{+}$transition, having independently verified the circular polarization of the $18190.43 \mathrm{~cm}^{-1}(\sim 550 \mathrm{~nm})$ laser beam required for this transition. The third step of the excitation sequence was driven by a laser tunable in the range $12050-11500 \mathrm{~cm}^{-1}$ $(\sim 830-840 \mathrm{~nm})$ which was also circularly polarized.

To decelerate the $\left|M_{J}\right|=3$ Rydberg-Stark states of $\mathrm{H}_{2}$ from their initial longitudinal velocity of $500 \mathrm{~m} / \mathrm{s}$ to zero velocity in the laboratory frame, pulsed high potentials $\left(\mp V_{\text {decel }}\right)$ are applied to electrodes 3 and 4 following photoexcitation. After a fast rise (30 ns) to their maximal value, the potentials decay exponentially with a time constant of $3.65 \mu \mathrm{s}$. This guarantees that the decelerating molecules experience a maximal field gradient throughout their trajectories while never being subject to a field of sufficient strength to cause ionization. At the end of the exponential decay, the potentials on electrodes 3 and 4 return to their initial values, and the center of the stationary Rydberg molecule cloud is located at the minimum of the three-dimensional trap (see Ref. [16] for additional details). This single-stage deceleration procedure minimizes transverse losses during deceleration and trap loading.

At any time after photoexcitation $\left(t_{\text {ion }}\right)$, a large positive electric potential of $+4 \mathrm{kV}$ can be switched on electrodes 1 and 2 to field ionize the Rydberg molecules and extract the $\mathrm{H}_{2}{ }^{+}$ions through a short flight tube toward a microchannel plate (MCP) detector. Analysis of the $\mathrm{H}_{2}{ }^{+}$time-of-flight (TOF) profiles allows the particle number in the decelerator and trap to be monitored along with the longitudinal position of the Rydberg molecule cloud [16,27]. The neutral Rydberg molecules can also be detected directly on the MCP detector if left to propagate through the flight tube without ionization.

A spectrum of the field-free $\left|M_{J}\right|=3 \mathrm{H}_{2}$ Rydberg states, measured by pulsed electric field ionization (PFI) 
at $t_{\text {ion }}=3 \mu \mathrm{s}$, is presented in Fig. 2(a). This spectrum includes states in the range $n=21-35$ of the $N^{+}=0$ series along with the $n=16-20$ states of the $N^{+}=2$ series. Figure 2(b) represents a Stark spectrum, also recorded by PFI, but with $V_{\text {trap }}=20 \mathrm{~V}$, resulting in an electric field at the excitation position of $56 \mathrm{~V} / \mathrm{cm}$. At this field, adjacent Stark manifolds start to overlap in energy at $n=31$. To assist in the identification of the long-lived states, a second spectrum [Fig. 2(c)] was recorded in the same excitation field but by detecting the neutral Rydberg molecules, excited in a pure $\mathrm{H}_{2}$ beam, when they impinge on the MCP detector after a TOF of $90 \mu$ s. Unlike the $N^{+}=2$ series, states of the $N^{+}=0$ series are sufficiently long-lived to permit detection following deceleration and trapping.

A spectrum containing only the long-lived $\left|M_{J}\right|=3$ Rydberg-Stark states that are accepted into the trap when $V_{\text {trap }}=20 \mathrm{~V}$ and $V_{\text {decel }}=1.7 \mathrm{kV}$, and remain trapped until detection by PFI at $t_{\text {ion }}=50 \mu \mathrm{s}$, is presented in Fig. 2(d). As the undecelerated beam of $\mathrm{H}_{2}$ molecules leaves the PFI region between electrodes 1-4 in less than $8 \mu \mathrm{s}$, all molecules detected by PFI after $50 \mu \mathrm{s}$ must have been trapped. Additionally, the TOF distributions of the $\mathrm{H}_{2}{ }^{+}$ions to the MCP detector following PFI (not shown) reveal that, beyond $25 \mu \mathrm{s}$ after excitation, the molecules are ionized from the same narrow spatial region around the trap minimum. The observation that the trapped states lie to the high-energy side of the zero-field resonances (vertical dashed lines) clearly indicates that only low-fieldseeking states are trapped. The deceleration and the acceptance of the trap depend upon the dipole moment, which scales as the product $n k$, and states of many different principal quantum numbers can be trapped because a de-

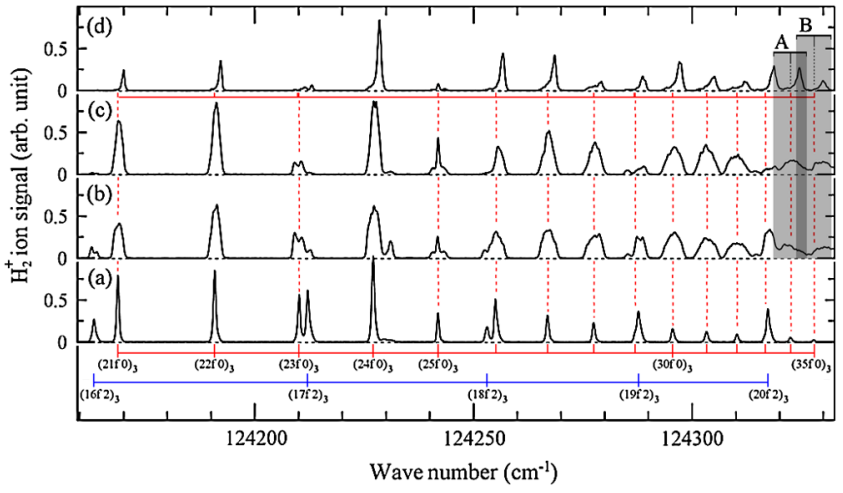

FIG. 2 (color online). (a) Field-free spectrum of $\ell=3,\left|M_{J}\right|=$ 3 Rydberg states of $\mathrm{H}_{2}$. (b) $\left|M_{J}\right|=3$ Stark spectrum recorded in an electric field of $56 \mathrm{~V} / \mathrm{cm}$, with PFI at $t_{\text {ion }}=3 \mu \mathrm{s}$. (c) Stark spectrum of long-lived $\left|M_{J}\right|=3$ states recorded by measuring the signal when the neutral molecules impinged on the MCP detector $90 \mu \mathrm{s}$ after photoexcitation in a field of $56 \mathrm{~V} / \mathrm{cm}$. (d) Low-field-seeking $\left|M_{J}\right|=3$ Rydberg-Stark states recorded by PFI following excitation in the same electric field of $56 \mathrm{~V} / \mathrm{cm}$ and deceleration and trapping for a time of $50 \mu$ s (see text for details). The field-free states are labeled $\left(n \ell N^{+}\right)_{J=\left|M_{J}\right|}$, and their positions are indicated by vertical dashed lines. crease in $n$ (or $k$ ) can be compensated by an increase in $k$ (or $n$ ). The two shaded regions on the right-hand side of the figure labeled $A$ and $B$ correspond to the energy ranges covered by the Stark manifolds of the $n=34$ and $n=35$ states of the $N^{+}=0$ series, respectively. The peaks corresponding to the trapped molecules in Fig. 2(d) are located in the region where the two Stark manifolds overlap, i.e., beyond the Inglis-Teller limit. Consequently, all avoided crossings encountered during deceleration and trap loading must have been traversed diabatically.

A demonstration of Stark-state-selective trap loading is presented in Fig. 3. In Fig. 3(a), the field-free $\left|M_{J}\right|=3$, $n=22$ line of the $N^{+}=0$ series recorded by PFI at $t_{\text {ion }}=$ $3 \mu \mathrm{s}$ is presented. In Fig. 3(b), the corresponding Stark manifold, recorded at the same ionization delay, but with $V_{\text {trap }}=100 \mathrm{~V}$, such that the electric field at the excitation position was $278 \mathrm{~V} / \mathrm{cm}$, is displayed. In this spectrum, the partially resolved Stark states are labeled by their quantum number $k$, which ranges from $k=-\left(n-\left|M_{J}\right|-1\right)$ to $k=$ $+\left(n-\left|M_{J}\right|-1\right)$ in steps of 2. Recording the same spectrum after deceleration with $V_{\text {decel }}=1.7 \mathrm{kV}$ and trapping for $50 \mu \mathrm{s}$, only the outer low-field-seeking Stark states with $k=10-18$ are observed [see Fig. 3(c)]. This is in agreement with numerical simulations of the Stark state acceptance of the decelerator and trap performed using the procedure described in Ref. [16]. Upon increasing the deceleration potential to $V_{\text {decel }}=2.3 \mathrm{kV}$, the acceptance of states with smaller dipoles increases, as is seen in Fig. 3(d), where the intensity of the $k=10$ and $k=12$ states is significantly enhanced compared to Fig. 3(c).

The rate of loss of the Rydberg $\mathrm{H}_{2}$ molecules from the trap has been studied by monitoring the ion signal as a function of the time between photoexcitation and PFI and

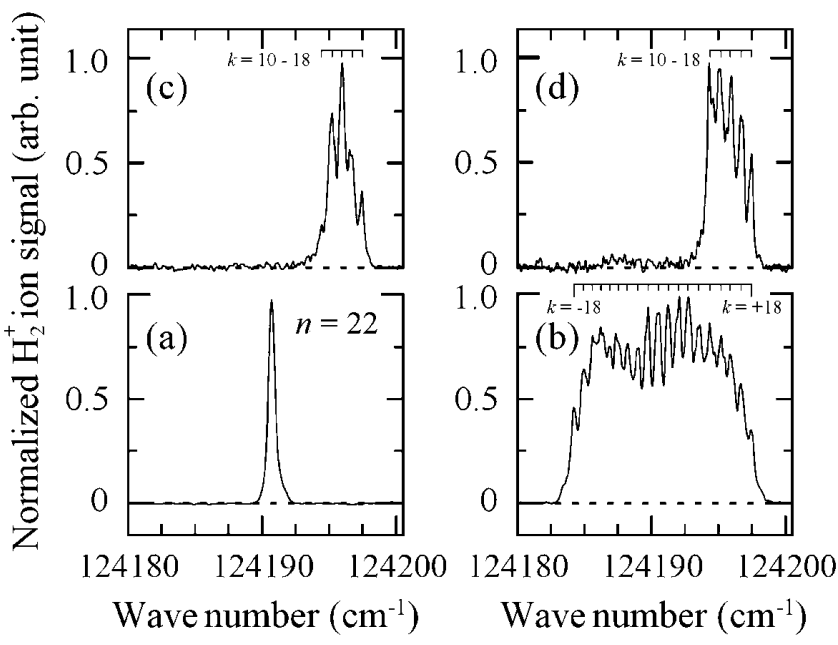

FIG. 3. (a) Field-free $n=22, \ell=3,\left|M_{J}\right|=3$ Rydberg state of $\mathrm{H}_{2}$ detected at $t_{\text {ion }}=3 \mu \mathrm{s}$. (b) $n=22,\left|M_{J}\right|=3$ Stark manifold recorded in an electric field of $278 \mathrm{~V} / \mathrm{cm}$ with detection at $t_{\text {ion }}=3 \mu \mathrm{s}$. Electrostatically trapped low-field-seeking $k=10-18, n=22,\left|M_{J}\right|=3$ Stark states of $\mathrm{H}_{2}$ detected at $t_{\text {ion }}=50 \mu \mathrm{s}$ with pulsed potentials of (c) 1.7 and (d) $2.3 \mathrm{kV}$ applied for deceleration. 


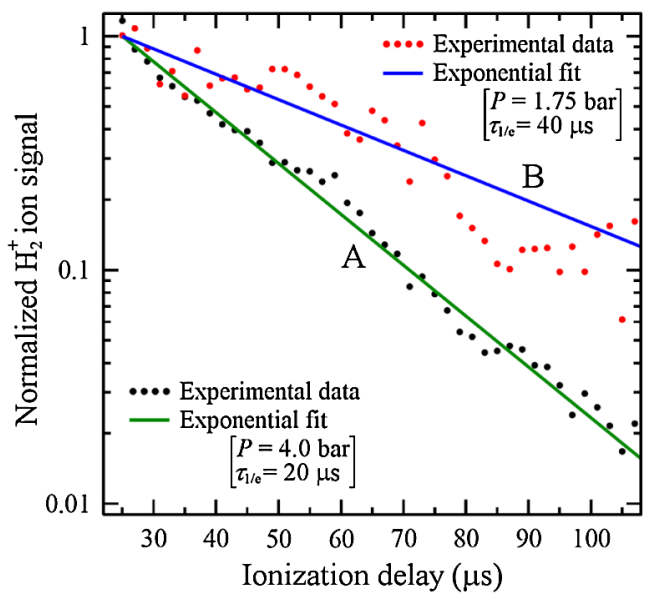

FIG. 4 (color online). Measurement of the $\mathrm{H}_{2}{ }^{+}$ion signal detected from the trap volume as a function of the delay between photoexcitation to the $n=33, k=23$ Stark state of the $N^{+}=0$ series and PFI. The data were recorded at an absolute $\mathrm{H}_{2}: \mathrm{Kr}$ stagnation pressure of (A) 4.0 and (B) 1.75 bar. The scatter of the data reflects the experimental uncertainties.

found to be strongly dependent on the stagnation pressure at which the pulsed valve was operated, as illustrated in Fig. 4. The number of $\mathrm{H}_{2}{ }^{+}$ions detected by PFI up to $115 \mu$ s after photoexcitation of the $n=33, k=23$ Stark state of the $N^{+}=0$ series decays exponentially with a time constant of $\tau_{1 / e}=20 \mu \mathrm{s}$ when the pulsed valve is operated at 4.0 bar (data set A). However, if the stagnation pressure is reduced to $1.75 \mathrm{bar}$, this time increases by a factor of $\sim 2$ to $\tau_{1 / e}=40 \mu \mathrm{s}$ (data set B), but the magnitude of the signal is also reduced because the lower density of the molecular beam leads to a smaller number of initially trapped molecules. These observations indicate that the measured trap decay times are limited by collisional processes. It is estimated that the trap is loaded at an initial density of $10^{7}-10^{8} \mathrm{~cm}^{-3}$ and a temperature of $\sim 150 \mathrm{mK}$, corresponding to a loading efficiency of $\sim 60 \%$.

Three-dimensional electrostatic trapping of cold molecular hydrogen has been demonstrated for the first time. Essential for the success of these experiments was the excitation of core-nonpenetrating Rydberg-Stark states in a multiphoton excitation scheme involving circularly polarized laser radiation. As all atoms and molecules possess Rydberg states, which can efficiently be deexcited to the absolute ground state, e.g., by the excitation sequence in reverse, the methodologies presented here provide a general route to the preparation of cold stationary samples of a wide range of atoms and molecules.

This work is supported by the Swiss National Science Foundation under Project No. 200021-113886 and by the European Research Council advanced grant program under Project No. 228286.
[1] M. T. Bell and T. P. Softley, Mol. Phys. 107, 99 (2009).

[2] S. Y.T. van de Meerakker, H. L. Bethlem, and G. Meijer, Nature Phys. 4, 595 (2008).

[3] L. D. Carr, D. DeMille, R. V. Krems, and J. Ye, New J. Phys. 11, 055049 (2009).

[4] B. Friedrich and J. M. Doyle, Chem. Phys. Chem. 10, 604 (2009).

[5] J. J. Gilijamse et al., Science 313, 1617 (2006).

[6] S. Willitsch, M. T. Bell, A. D. Gingell, S. R. Procter, and T. P. Softley, Phys. Rev. Lett. 100, 043203 (2008).

[7] P. F. Staanum, K. Højbjerre, R. Wester, and M. Drewsen, Phys. Rev. Lett. 100, 243003 (2008).

[8] M. R. Tarbutt et al., Phys. Rev. Lett. 92, 173002 (2004).

[9] J. van Veldhoven et al., Eur. Phys. J. D 31, 337 (2004).

[10] A. Fioretti et al., Phys. Rev. Lett. 80, 4402 (1998).

[11] J. M. Doyle, B. Friedrich, J. Kim, and D. Patterson, Phys. Rev. A 52, R2515 (1995).

[12] H. L. Bethlem, G. Berden, and G. Meijer, Phys. Rev. Lett. 83, 1558 (1999).

[13] N. Vanhaecke, U. Meier, M. Andrist, B. H. Meier, and F. Merkt, Phys. Rev. A 75, 031402(R) (2007).

[14] E. Narevicius et al., Phys. Rev. A 77, 051401(R) (2008).

[15] H. L. Bethlem et al., Nature (London) 406, 491 (2000).

[16] S. D. Hogan and F. Merkt, Phys. Rev. Lett. 100, 043001 (2008).

[17] J. van Veldhoven, H.L. Bethlem, and G. Meijer, Phys. Rev. Lett. 94, 083001 (2005).

[18] B. C. Sawyer et al., Phys. Rev. Lett. 98, 253002 (2007).

[19] S. D. Hogan, A. W. Wiederkehr, H. Schmutz, and F. Merkt, Phys. Rev. Lett. 101, 143001 (2008).

[20] T. Breeden and H. Metcalf, Phys. Rev. Lett. 47, 1726 (1981).

[21] W. H. Wing, Phys. Rev. Lett. 45, 631 (1980).

[22] D. Townsend, A. L. Goodgame, S. R. Procter, S. R. Mackenzie, and T.P. Softley, J. Phys. B 34, 439 (2001).

[23] S. R. Procter, Y. Yamakita, F. Merkt, and T. P. Softley, Chem. Phys. Lett. 374, 667 (2003).

[24] Y. Yamakita, S. R. Procter, A. L. Goodgame, T. P. Softley, and F. Merkt, J. Chem. Phys. 121, 1419 (2004).

[25] E. Vliegen, H. J. Wörner, T. P. Softley, and F. Merkt, Phys. Rev. Lett. 92, 033005 (2004).

[26] E. Vliegen and F. Merkt, J. Phys. B 38, 1623 (2005).

[27] E. Vliegen and F. Merkt, Phys. Rev. Lett. 97, 033002 (2006).

[28] E. Vliegen, S. D. Hogan, H. Schmutz, and F. Merkt, Phys. Rev. A 76, 023405 (2007).

[29] A. Kellerbauer et al., Nucl. Instrum. Methods Phys. Res., Sect. B 266, 351 (2008).

[30] T. C. Killian et al., Phys. Rev. Lett. 83, 4776 (1999).

[31] M. D. Lukin et al., Phys. Rev. Lett. 87, 037901 (2001).

[32] T.F. Gallagher, Rydberg Atoms (Cambridge University Press, Cambridge, England, 1994).

[33] F. Merkt, Annu. Rev. Phys. Chem. 48, 675 (1997).

[34] A. Osterwalder, A. Wüest, F. Merkt, and Ch. Jungen, J. Chem. Phys. 121, 11810 (2004). 